\title{
Functional Outcome of Titanium Elastic Nailing in Pediatric Tibial Fracture
}

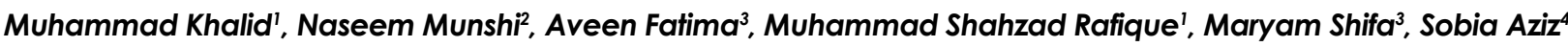
'Bantva Memon Hospital, Karachi, 'Ziauddin Hospital North Nazimabad, ${ }^{3}$ Student, "4Department of General Surgery, Dr. Ziauddin Hospital, Karachi, Pakistan.

\begin{abstract}
Background: Tibial Diaphyseal fracture (TDF) is the third most common fracture seen in the pediatric population. Titanium elastic nailing (TEN) has been ideal for the management of tibial diaphyseal fractures to prevent complications. Therefore, this study aimed to access the functional outcome of TEN in pediatric Tibial diaphyseal fracture.

Methods: This prospective cohort study was conducted on $\mathrm{n}=87$ skeletally immature patients (6-12 years) at the two hospitals in Karachi from $1^{\text {st }}$ November 2019-30 th April 2021 including patients having unilateral, closed Tibial Diaphyseal fracture. The 75 patients ( 12 of them were lost follow-up) were analyzed in terms of wound infection, union, weight-bearing, limb shortening, rotation, angulation, and range of movement at knee and ankle joints. Paired t-test was used to compare the variables with a $p$-value $<0.05$ was considered statistically significant.
\end{abstract}

Results: Patients $\mathrm{n}=75$ in this study had a mean age of $8.32 \pm 1.92$ years with a mean weight of 25.8 \pm 6.9. The most frequent cause of injury was traffic accident $49(65.3 \%)$, followed by falling $19(25.3 \%)$ and $7(9.4 \%)$ with other causes. The most common fracture type was transverse $45(60 \%)$, followed by oblique $26(34.7 \%)$, and the remaining $4(5.3 \%)$ had a spiral fracture. All patients were allowed full weight-bearing at $9.8 \pm 1.5$ week. At the end of this study, all patients regained knee and ankle range of motion $(p<0.0001)$.

Conclusion: Titanium elastic nailing was the ideal procedure for Tibial Diaphyseal fracture in the pediatric population, resulting in a short hospital stay, a rapid rehabilitation without complications.

Keywords: Pediatrics; Pediatric Tibial Diaphyseal Fracture; Titanium; Titanium Elastic Nailing; Functional Outcome; Movement.

\author{
Corresponding author: \\ Dr. Muhammad Khalid \\ Bantva Memon Hospital, \\ Karachi, Pakistan. \\ Email: drmkarain@gmail.com \\ https://doi.org/10.36283/PJMD1 1-1/003
}

How to cite: Khalid M, Munshi N, Fatima A, Rafique MS, Shifa M, Aziz S. Functional Outcome of Titanium Elastic Nailing in Pediatric Tibial Fracture. 2022; 1 (1): 1 1-16.doi: 10.36283/PJMD1 1-1/003

\section{INTRODUCTION}

Pediatric tibial diaphyseal fracture (TDF) is the third most common fracture, contributing to $15 \%$ of all pediatric fractures ${ }^{1,2}$. Pediatric TDF, commonly found in young boys, are mostly oblique or transverse fractures of the middle and distal third of the shaft ${ }^{2,3}$. Thus, $70 \%$ of the fractures are isolated TDF, which are minimally displaced at presentation, 
while the remaining $30 \%$ are associated with Ipsilateral fibular fractures ${ }^{4,5}$. TDF in younger children is commonly caused due to minor falls and twisting injures. While in older children it is caused by sports-related trauma or motor vehicle accidents ${ }^{5,6}$.

Treatment is based upon a patient's age, concomitant injuries, and type of fracture ${ }^{5}$. Most TDF are treated with cast immobilization and closed reduction, however in cases where these methods do not provide an acceptable reduction in injures, surgical intervention such as external fixation and plate and screw fixation is used ${ }^{7,10}$. Over the last decade use of titanium elastic nailing (TEN) has been used to treat pediatric TDF to achieve optimum post-operative results with a low rate of complications ${ }^{9-11}$. TEN as compared to the external fixation offers minimal intrusion using small incisions and provides fewer chances of surgical trauma to the fracture site allowing early union and rehabilitation. External fixation is a heavyweight implant that might hinder the child's rehabilitation. Hence this method is not preferable in children ${ }^{10-12}$.

Intramedullary nailing can be of two types rigid or flexible. Although Rigid locked intramedullary nailing provides stable fixation, its use is avoided due to its tendency to damage proximal tibial physis. Hence elastic intramedullary nailing is preferable while treating TDF ${ }^{1-14}$. Closed reduction with percutaneous Kirschner wire fixation and cast immobilization is useful for unstable, oblique fractures in younger children ${ }^{11,12}$.

The main objective of this study was to assess the functional and radiological outcome of the TEN in the pediatric age group. Many studies have been conducted on this topic internationally however, in Pakistan not much data is available. Hence this study will be extremely helpful in discovering new treatment options for pediatric TDF. This study will not only give standard guidelines to surgeons regarding treatment but will also ensure the patients' mental and emotional wellbeing through quick and early mobilization and a complication-free treatment.

\section{METHODS}

This prospective cohort research was carried out in the Orthopedic Department of Bantva Memon Hospital, Kharadar and Ziauddin Hospital North Nazimabad campus, Karachi. Using the purposive sampling technique, 87 skeletally immature patients, aged 6-12 years, were selected having unilateral, closed TDF. All patients were admitted through the accident, emergency, and outpatient departments. The duration of the study was 18 months from $1^{\text {st }}$ November 2019 to 30 $30^{\text {th }}$ April 2021. Each patient was followed up for 1 year. All patients with polytrauma, pathological fracture, or previous surgery of limb were excluded from this study. The study was approved by the hospital ethical committee of Bantva Memon Hospital.

All selected patients were clinically examined and radiologically investigated to confirm the diagnosis. Preoperative routine investigations were done. From March 2020 onwards any patient admitted to the hospital for surgery was required to take a PCR Covid-19 test. Patients were explained about the risks and surgical outcome and informed consent were taken. Demographic and preoperative data were collected in Performa which included, age, sex, weight, fracture type, fracture side, cause of injury, and time duration between trauma to surgery.

During the TEN procedures, all patients were placed in a supine position under general anesthesia. Closed reduction was attempted after adequate reduction was achieved two nails with diameter 1/3 to that of the intramedullary canal were implanted through small incisions on lateral and medial sides of proximal tibia metaphysis. The nails were advanced beyond the fracture site bent slightly away from the bone to give stability. Before wound closure was performed, the accuracy of closed reduction was evaluated using the C-arm image intensifier intraoperatively. After three unsuccessful attempts at closed reduction, open reduction was performed with a small lateral incision followed by two Titanium elastic nails to stabilize the fracture.

To maintain stability after the surgery, an above-knee cast was applied for six weeks. Follow-up visits were made at 2, 6, 12, 24 weeks, and then every 3 months for one year. On the $2^{\text {nd }}$ week follow up the Plaster of Paris back slab was removed, the wound was examined, the stitches were removed and the cast was reapplied for further four weeks. On the $6^{\text {th }}$ week follow up the cast was removed and partial weight-bearing was started. Physical therapy was started for initial gait training, more active exercises were started after the callus had reappeared. 6-8 months after the surgery, nails were removed when the fracture line was no longer visible radiologically.

At each postoperative follow-up visit, clinical and radiological assessments were done. The outcome of the data collection includes mean hospitalization stay, full weight-bearing time, rate of open reduc- 
tion, fracture union, and postoperative complications comprising of nail discomfort, broken nails, infected wounds, limb shortening/lengthening, and delayed or nonunion. Final functional results were assessed according to the modified Hindley score.

The collected data will be entered into SPSS version 23. Quantitative variables, such as age, will be analyzed in terms of mean \pm standard deviation. Qualitative variables such as gender will be documented in terms of frequency and percentage. Paired t-test was used to compare the variables with a $p$ value < 0.05 was considered statistically significant.

\section{RESULTS}

Patients $(n=87)$ were selected based on inclusion criteria and were operated on, 12 of whom lost follow-up. The final data were collected from 75 patients, 56 (74.7\%) were boys and 19 (25.3\%) were girls. The average age was $8.32 \pm 1.92$. The average weight of the patients was $25.8 \pm$ 6.9. The most frequent cause of injury was a traffic accident, which accounted for 49 patients (65.3\%), 19 patients had a history of falling (25.3\%). The remaining 7 patients had other causes (9.4\%). The most common fracture type was transverse, having 45 patients $(60 \%)$, then was oblique with 26 patients $(34.7 \%$ ) and the remaining 4 patients (5.3\%) had a spiral fracture. 43 (57.3\%) patients had a left tibia fracture and $32(42.7 \%)$ had a right tibia fracture (Figure 1, Table 1). The average time from accident to operation was $5 \pm 1$ days. The mean hospitalization time was $3 \pm 1$ days. In 75(100\%) patients closed reduction was successful in the first attempt, while in the other $4(5.4 \%)$ reduction was achieved in the second attempt. None of our patients were treated through open reduction. The fracture union time was $8.6 \pm 2$ weeks. The full weight-bearing time was $9.8 \pm 1.5$ weeks.

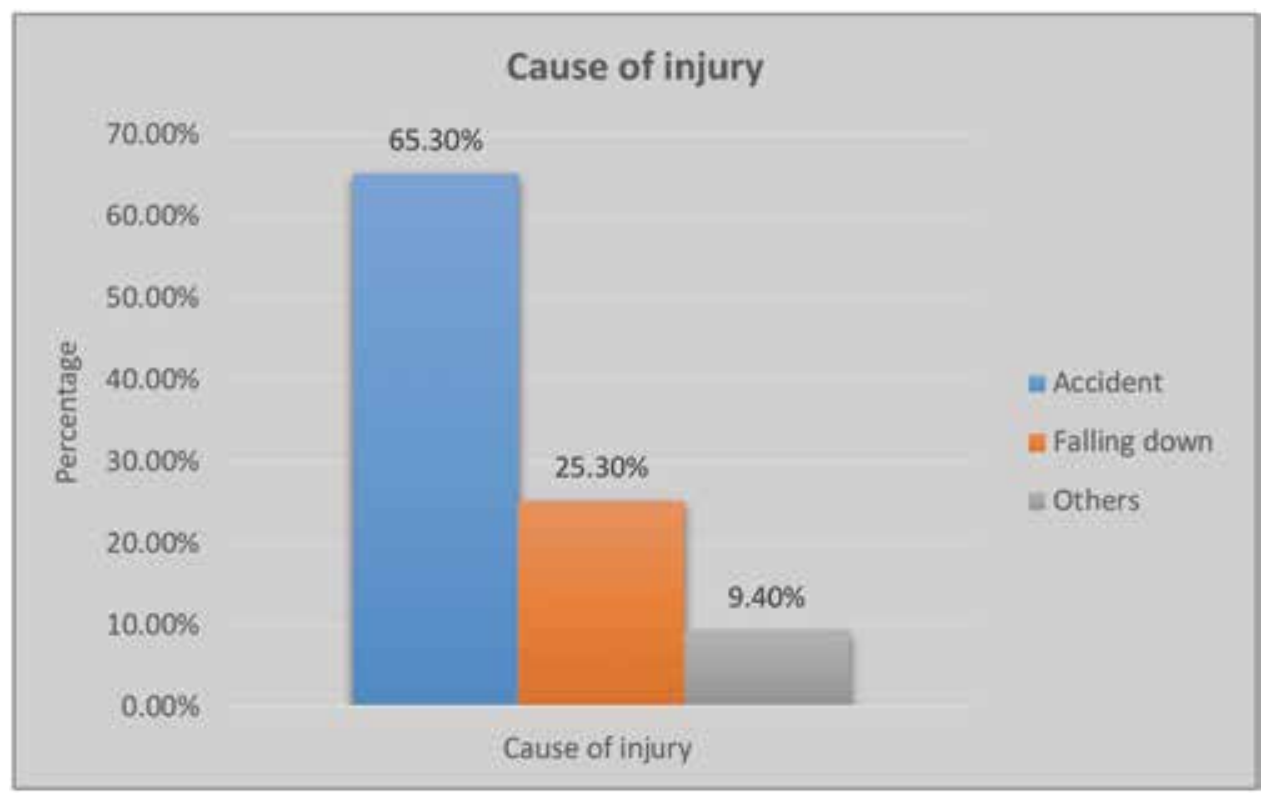

Figure 1: Causes of injury among the patients.

Table 1: Radiological assessment of fracture union.

\begin{tabular}{|l|c|c|c|c|}
\hline Follow up & Patients & Cortical Scoring & Callus Formation & Fracture Line \\
\hline 2 -week & $75(100 \%)$ & 1 & Absent & Visible \\
\hline \multirow{3}{*}{6 -week } & $54(72 \%)$ & 2 & Present & Visible \\
\cline { 2 - 5 } & $2(12.6 \%)$ & 1 & Absent & Visible \\
\cline { 2 - 5 } & $19(25.3 \%)$ & 3 & Bridging & Visible \\
\hline \multirow{3}{*}{12 -week } & $66(88 \%)$ & 3 & Present & Visible \\
\cline { 2 - 6 } & $3(4 \%)$ & 2 & Remodeling & Not visible \\
\cline { 2 - 6 } & $6(8 \%)$ & 4 & Remodeling & Not visible \\
\hline
\end{tabular}


Follow up period time was an average of $21.27 \pm$ 6.70 months. During the postoperative follow-up visit, there was no case of delayed, nonunion, or nail breakage. Superficial infection was seen only in 2 patients $(2.7 \%)$ who were treated successfully. Thus, 4 patients (5.3\%) experienced nail irritation, which subsided without any treatment. There were 2 cases of limb shortening less than $2 \mathrm{~cm}$. These cases come under satisfactory outcomes. There was no internal or external rotation found in any of the cases (Figure 2). The anterior or posterior angulation found in 6 patients was below $10^{\circ}$, and medial and lateral rotations were less than $5^{\circ}$ seen in 4 patients, which according to the protocol were good (Table 2). The movements of knee and ankle joints were within normal limits except in 3 cases, where the range of knee motion was at $60 \%$ and after physiotherapy became $85 \%(p=<0.0001$, Table 3$)$.

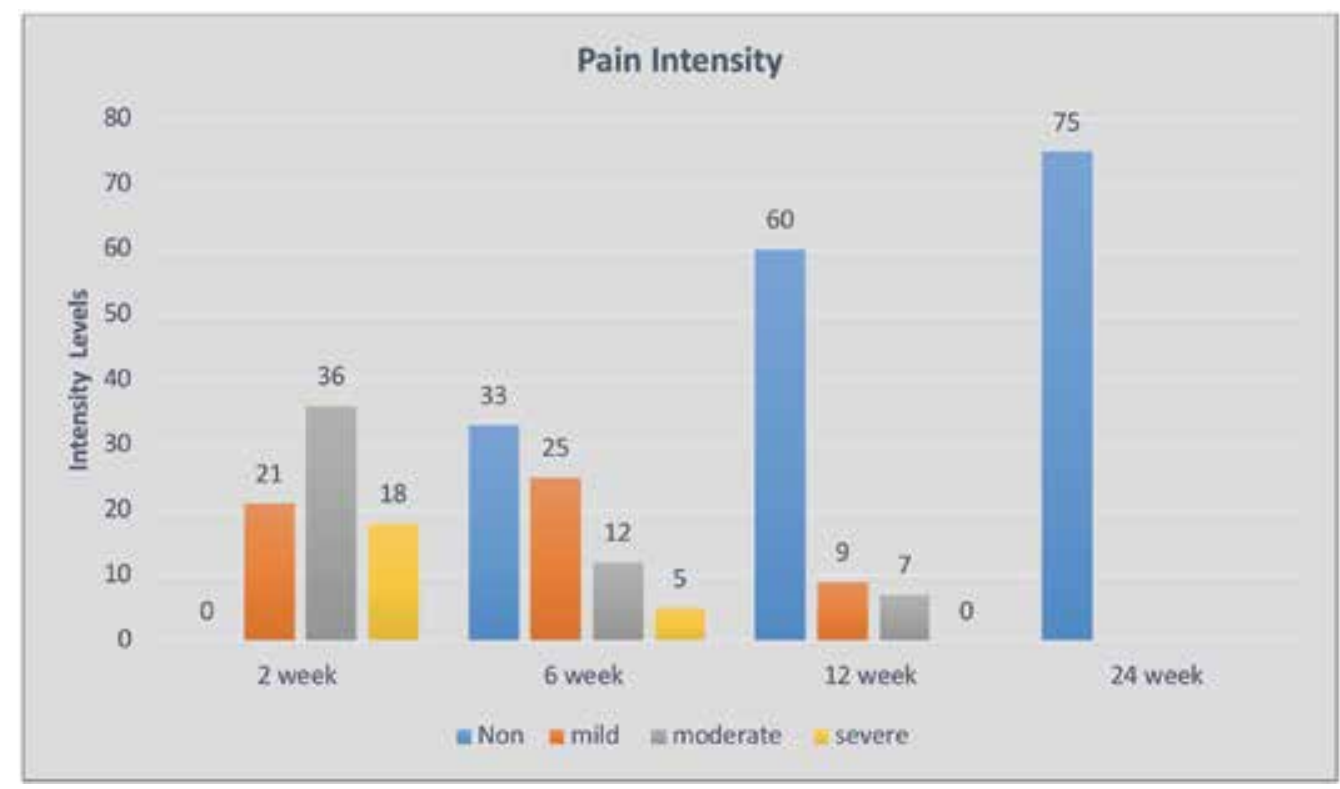

Figure 2: Pain intensity on follow-up visits.

Table 2: Comparison of range of motion at the knee joint

\begin{tabular}{|l|c|c|c|c|c|c|}
\hline Range of motion & \multicolumn{3}{|c|}{ Extension at Knee Joint } & \multicolumn{3}{c|}{ Flexion at Knee Joint } \\
\hline Follow up visit & 6-week & 12 -week & 24 -week & 6-week & 12 -week & 24 -week \\
\hline Mean \pm SD & $21.27 \pm 6.70$ & $8.87 \pm 4.90$ & $2.13 \pm 2.70$ & $89.04 \pm 6.48$ & $115.28 \pm 4.41$ & $129.32 \pm 4.40$ \\
\hline $\mathrm{p}$-Value & $<0.0001$ & $<0.0001$ & $<0.0001$ & $<0.0001$ & $<0.0001$ & $<0.0001$ \\
\hline
\end{tabular}

*Paired t-test applied for repeated observations.

Table 3: Functional outcome on the final follow-up visit.

\begin{tabular}{|l|c|c|}
\hline Complications & Excellent & Good \\
\hline Valgus/ Varus & $71(94.6 \%)$ & $4(5.4 \%)$ \\
\hline Anteversion/ Recurvatum & $69(92 \%)$ & $6(8 \%)$ \\
\hline Superficial Infection & $73(97.3 \%)$ & $2(2.7 \%)$ \\
\hline Limb Shortening & $73(97.3 \%)$ & $2(2.7 \%)$ \\
\hline Knee Motion & $72(96 \%)$ & $3(4 \%)$ \\
\hline Rotation & $75(100 \%)$ & $0(0 \%)$ \\
\hline Nonunion/ Delayed union & $75(100 \%)$ & $0(0 \%)$ \\
\hline
\end{tabular}

\section{DISCUSSION}

TDF is the third most common type of pediatric fracture. For decades, cast immobilization has been a standard method for TDF treatment ${ }^{15,16}$. The basic treatment principles of TDF are based upon the restoration of bone alignment, early mobilization, and rehabilitation after the union ${ }^{17}$. The invention of TEN made a revolution in the management of pediatric
TDF. This is a biomechanically ideal method for weight-bearing long bone ${ }^{18-20}$. The current study showed excellent results, like studies conducted previously. The average time for fracture union is $8.6 \pm$ 2 weeks. The full weight-bearing time was $9.8 \pm 1.5$ weeks. During the postoperative follow-up visit, there was no case of delayed, nonunion, or nail breakage. Knee and Ankle movements were satisfactory. There was no rotational deformity seen. This study shows good functional outcomes due to quick fracture union, rapid recovery, and rehabilitation.

The results of numerous studies show TEN is the ideal method to treat TDF in children, giving the best results. Economedes et al., reported 19 TDF patients, all of whom achieved bony union at an average of 11 weeks. Five of these patients (26\%) experienced irritation at the nail entry site ${ }^{11}$. 
Onta et al., studied 18 children, having an average age of 8.2 years, with TDF. The average fracture healing time was 13.3 weeks. 16 children had an angulation of less than $5^{\circ}$ and 2 patients had an angulation between $5-10^{\circ}$, which was considered acceptable for their age ${ }^{19}$. Hindley conducted a study of 6-month duration, on 22 patients. 18 patients had an excellent result and the remaining four patients had a satisfactory result ${ }^{13}$. Kamran et al., studied 35 patients all of whom achieved acceptable movement at knee and Ankle joints with no complications ${ }^{8}$. Furlan studied 175 patients, 36 of whom were TDF, which were treated with TEN. ThUs, $89 \%$ of them had an excellent result, $11 \%$ was satisfactory and none of the patients were dissatisfied with their treatment and results ${ }^{20}$. Kubiak et al conducted a study comparing TEN to external fixation with patients having open and closed TDF. In this study, a significant decrease in the fracture union time was reported in TEN as compared to external fixation ${ }^{21}$. Furthermore, superficial pin tract infections were seen in some of the patients with open breaks, and these patients were treated with anti-biotherapy with no requirement for any extra mediations $^{22}$. In our facility, we typically leave the TEN finishes on top of the skin because of the restricted delicate tissue support in the proximal tibia, which could clarify the event of the pin tract diseases in some of them and no different patients in comparative examinations $^{23}$. Hence, a second intramedullary TEN with a fitting distance across was embedded through the proximal average of the tibia ${ }^{24}$. Following the assertion of fracture reduction and the placing of the TENs with C-arm ${ }^{25}$.

\section{CONCLUSION}

Titanium elastic nailing TEN is the treatment of choice for pediatric Tibial Diaphyseal fracture (TDF). It is a minimally invasive technique that ensures early and complete mobilization with a short hospitalization stay. This technique has a minimum complication rate and early rehabilitation which ensures a quicker return to school and their normal routine life is the third most common fracture seen in the pediatric population.

\section{ACKNOWLEDGEMENT}

We would like to acknowledge the hospital staff, OT technicians, nurses, and physiotherapists for their co-cooperation and contribution to this study.

\section{CONFLICT OF INTEREST}

The authors declared no conflict of interest.

\section{ETHICS APPROVAL}

The study was approved by ethical committee of Bantva Memon Hospital.

\section{PATIENT CONSENT}

Written consents were taken for participation before the start of the study.

\section{AUTHORS' CONTRIBUTION}

MK analyzed and interpreted the patients' data and constructed the study design. NM analyzed and interpreted the patients' data and proofread the final article. AF was the major contributor in writing the manuscript MSR carried out the data entry and statistical analysis MS sorted out the references and points for writing the discussion section. SA also assisted in data entry and statistical analysis.

\section{REFERENCES}

1. Egol KA, Koval KJ, Zuckerman JD. Handbook of fractures. Lippincott Williams \& Wilkins; 2010, p. 3.

2. Patel NK, Horstman J, Kuester V, Sambandam S, Mounasamy $\vee$. Pediatric tibial shaft fractures. Indian J Orthop. 2018;52(5):522-528. doi: 10.4103/ortho.IJOrt ho_486_17

3. Setter KJ, Palomino KE. Pediatric tibia fractures: current concepts. Curr Opin Pediatr. 2006;18(1):30-35. doi: 10.1097/01.mop.0000192520.48411.fa

4. Mashru RP, Herman MJ, Pizzutillo PD. Tibial shaft fractures in children and adolescents. J Am Acad Orthop Surg. 2005; 13:345-352.

5. Abdelbaset A, Fawaz KH. Treatment of tibial fractures in children by flexible intramedullary nails. Egypt Orthop J. 2018, 54 (2): 85-92.

6. Zeng S, Deng H, Zhu T, Han S, Xiong Z, Tang S. Calcaneal skeletal traction versus elastic intramedullary nailing of displaced tibial shaft fractures in children. Injury. 2021;52(4):849-854. doi: 10.1016/j.injury.2020.10.035

7. Fernandez FF, Egenolf $M$, Carsten C, Holz F, Schneider S, Wentzensen A. Unstable diaphyseal fractures of both bones of the forearm in children: Plate fixation versus intramedullary nailing. Injury. 2005; 36:1210-1216. doi: 10.1016/j.injury.2005.03.004 8. Kamran B, labal MZ, Rauf AC. Outcome of closed titanium elastic nailing in pediatric tibial diaphyseal fractures. J Sheikh Zayed Med Coll. 2016; 7(4):10741077.

9. Khuntia S, Swaroop S, Patro BP, Sahu S. Paediatric long bone fractures managed with elastic intramedullary nails: A retrospective study of 30 patients. Cureus. 2020;12(4):1-10. doi: 10.7759/cureus.7847

10. KC KM, Acharya $P$, Sigdel A. Titanium elastic nailing system (TENS) for tibia fractures in children: Functional outcomes and complications. J Nepal Med Assoc. 2016;55(204):55-60.

11. Economedes DM, Abzug JM, Paryavi E, Herman MJ. Outcomes using titanium elastic nails for open and closed pediatric tibia fractures. Orthopedics. 2014; 37:619-624. doi: 10.3928/01477447-20140626-52

12. Sahu RL, Ranjan R. Fracture union in percutaneous kirschner wire fixation in paediatrictibial shaft fractures. Chinese journal of traumatology. 2016; 19:353-357. doi: 10.1016/j.cjtee.2016.08.003

13. Hindley CJ. Closed medullary nailing for recent fractures of the tibia. Injury. 1988; 19(3):180-184. doi: 10.1016/0020-1383(88)90011-3 
14. Meena D, Tiwari AK. Results of management of tibia shaft fractures in children with titanium elastic nailing. Int J Orthop Sci. 2019; 5(4):878-881. doi: 10.22271/ortho.2019.v5.i4o.1789

15. Shen K, Cai H, Wang Z, Xu Y. Elastic stable intramedullary nailing for severely displaced distal tibial fractures in children. Medicine. 2016;95(39):1-6. doi: 10.1097/MD.0000000000004980

16. Pennock AT, Bastrom TP, Upasani VV. Elastic intramedullary nailing versus open reduction internal fixation of pediatric tibial shaft fractures. J Pediatr Orthop. 2017;37(7):403-408. doi: 10.1097/BPO.0000000000001065

17. Niazi MU, Shah SW, Qadeer R, Qadir A, Habib Z, Dahal $S$, et al. Comparison of casting versus elastic nail for the treatment of pediatric tibial diaphyseal fractures. Ann Pak Inst Med Sci. 2019;15(4):148-153.

18. Ghilley SK, Meena MK, Jhanwar P, Jain HK. Use of flexible intramedullary nailing in treating diaphyseal fractures of long bone of lower limb in children. J Orthop Traumatol Rehab. 2019;11:21-26. doi: 10.4103/jotr.jotr_5_19

19. Onta PR, Thapa P, Sapkota K, Ranjeet N, Kishore A, Gupta M. Outcome of diaphyseal fracture of tibia treated with flexible intramedullary nailing in pediatrics age group; a prospective study. Am J Public Health Res. 2015; 3(4A):65-68. doi:10.12691/ajphr-3-4A-14

20. Furlan D, Pogorelić Z, Biočić M, Jurić I, Budimir D,
Todorić J, et al. Elastic stable intramedullary nailing for pediatric long bone fractures: experience with 175 fractures. Scand J Urol. 2011; 100(3):208-215. doi: 10.1177/145749691110000313

21. Kubiak EN, Egol KA, Scher D, Wasserman B, Feldman D, Koval KJ. Operative treatment of tibial fractures in children: are elastic stable intramedullary nails an improvement over external fixation? JBJS. 2005;87(8):1761-1768. doi: 10.2106/JBJS.C.01616

22. Goodbody CM, Lee RJ, Flynn JM, Sankar WN. Titanium elastic nailing for pediatric tibia fractures: do older, heavier kids do worse? J Pediatr Orthop. 2016;36(5):472-477. doi: 10.1097/BPO.000000000000 0483

23. Uludağ A, Tosun HB. Treatment of unstable pediatric tibial shaft fractures with titanium elastic nails. Medicina. 2019;55(6):1-9. doi: 10.3390/medicina5506 0266

24. Moroz LA, Launay F, Kocher MS, Newton PO, Frick SL, Sponseller PD, et al. Titanium elastic nailing of fractures of the femur in children: predictors of complications and poor outcome. J Bone Joint Surg Br. 2006;88(10):1361-1366. doi: 10.1302/0301-620X.88B10. 17517

25. Sankar WN, Jones KJ, David Horn B, Wells L. Titanium elastic nails for pediatric tibial shaft fractures. J Child Orthop. 2007; 1 (5):281-286. doi: 10.1007/s1 1832 -007-0056-y 